rapidly. He considers that there were two epicentral zones, one directed north-north-east in the central part of Sagami Bay, the other intersecting it and directed north-west. The former coincides with the great region of subsidence in the bay, and this subsidence produced the condensational initial motion around the epicentre. Beneath the second epicentral zone, he thinks, there was a horizontal sliding of a layer of the crust towards the south-west, which would produce condensational motion on the south-west side and rarefactional motion on the north-east side. The vertical displacement beneath the first zone being the more important, the condensational initial motion prevailed at some distance from the origin. Among the secondary causes of the earthquake Mr. Suda includes the typhoon which was passing over the northern part of the epicentral region at the instant when the earthquake occurred.

C. D.

\title{
Italian Theses on Chemistry and Physiology.
}

THE twenty-sixth volume of reports, recently issued by the Cagnola Scientific Foundation, covers the period I9I7-I923 and deals with the essays on various scientific subjects submitted for adjudication to committees appointed by the Royal Lombardy Institute of Science and Literature. The subjects vary widely in character, but are all of medical or chemical interest. In a few instances only have the theses presented been deemed worthy of reward, and of the successful theses, four only are printed in extenso in the present volume.

In "The Chemio-therapy of the Spirilli," which extends to over I6o pages and is accompanied by many plates, diagrams, etc., Prof. Giorgio Castelli gives, in addition to a succinct account of previous knowledge of this subject, the results of his own numerous experiments, which lead to various important conclusions. The slight increase in the efficacy of the curative agents determined by prolonged contact of the latter with the air during the manipulation is insufficient to compensate for the dangerous increase often observed in the toxicity. Oral administration of the arsenobenzenes is not to be recommended, and the use of dimethylaminotetraminoarsenobenzene, proposed by Giemsa, is not only less effective than that of salvarsan or neosalvarsan, but is also followed by more frequent and more ready relapse. Increased toxicity is also against the employment of compounds obtained by the introduction of amino and other nitrogenous groupings, sulphur, etc., into the molecule of arsenobenzene. In some cases augmented therapeutic efficacy is attained with compounds of salvarsan with silver or copper salts, but here too the frequency of undesirable after-effects militates against the application of such compounds on a large scale.

Prof. Aldo Perroncito, in a short contribution on the derivation of blood platelets, demonstrates the possibility of the formation of new platelets independently of the elements of all other tissues of the organism. In blood drawn from the jugular vein of the cat or dog, and treated with sodium citrate to render it non-coagulable, and then with pyrodin solution, the number of the platelets becomes doubled or, in some instances, even quintupled after the blood has been kept at $38^{\circ} \mathrm{C}$. for about an hour. Exclusion of the intervention of either the corpuscles or the plasma leads to the conclusion that the platelets are capable of reproducing other elements with similar morphological characters.

In his memoir entitled "Catalytic and Enzymic Phenomena," occupying I 30 pages and including a very useful bibliography, Prof. Ugo Pratolongo develops a novel interpretation of recent results arising from modern mechanico-static theories of chemical phenomena. He has succeeded in distinguishing in chemical kinetic equations the terms depending on piezometric actions and on medial actions from terms proper to the mechanism of the reaction. The application of this principle to wellknown accelerating reactions shows that some of these are definitely catalytic in nature, inasmuch as a change in the mechanism of the chemical reaction, and hence in the critical energy relative to the reaction, is involved; others are of medial character, and others again-heterogeneous catalyses-possibly depend on variations in the active concentration of the reagents induced by the catalyst. The experimental results show that, in the decomposition of hydrogen peroxide, the critical energy assumes values depending strictly on the metal functioning as catalyst and independent both of its method of preparation or state of subdivision and also of the presence of activating or inactivating agents. Thermal inactivation of a catalyst consists of partial destruction of the catalyst, the remaining fraction retaining its activity unaltered.

Under the title "Interchange of Hæmoglobin in Physiological and Pathological Conditions," Dr. Enrico Greppi discusses the different factors operative in the continual destruction (hæmolysis) and reformation (hæmoglobinogenesis) of the blood-pigment.

\section{Astronomical Observations at Greenwich and the Cape.}

THE first impression on seeing the annual volume for I92I of "Greenwich Observations" 1 is its thinness in comparison with the volumes of two or three decades ago. It appears to be the least bulky in the last hundred years. This is due to a reduction accomplished during Sir Frank Dyson's tenure of office by the suppression of much of the detail of the observations, but enough is still given to show the data on which the tabulated observations depend.

The results are given concisely and orderly, and they represent an immense amount of routine work which is carried on from year to year at Greenwich, a work never in the limelight but, on its astronomical

1 Astronomical and Magnetical and Meteorological Observations made at the Royal Observatory, Greenwich, in the year $192 \mathrm{I}$. side at least, the basis for the discussion of all the problems of fundamental astronomy:

The observations with the Transit Circle take up the first section, and show the state of adjustment of the instrument from day to day, and then give the observations of right ascension and declination of the sun, moon, and planets, together with the observed errors of the tabular place as given in the Nautical Almanac. More than I7,0oo observations were made in connexion with the Transit Circle in the course of the year. The observations of the moon have been combined with the extra-meridian ones taken with the Altazimuth, and give the error both of the Nautical Almanac (Hansen's tables with Newcomb's corrections), and Delaunay (Connaissance des temps, 